\title{
Detection and Classification of Cancer on CT Lung Images using Krill Herd Optimization Algorithm and ANN
}

\author{
J. Maruthi Nagendra Prasad ${ }^{1}$, Dr.M.Vamsi Krishna² \\ ${ }^{1}$ Research Scholar, Department of CSE, Centurion University of Technology and Management, Paralakhemundi, Orissa, \\ India,maruthiprasad1986@gmail.com \\ ${ }^{2}$ Professor, Department of Computer Science and Engineering, Chaitanya Engineering College, Kakinada, \\ India,vamsimangalam@gmail.com
}

\begin{abstract}
The most terrifying type of cancer is lung cancer, which is treated as very common disease amongst humans. Now a days, earlier detection of lung cancer is mandatory in order to enhance the survival rate of the afflicted. Earlier detection of lung cancer is the area, which is under the radar of most of the researchers because with earlier detection the life expectancy of the afflicted will be improved. For the last few years more of the research is going on to predict the cancer at the beginning stages.Here in this work, the raw CT Lung images are used which are preprocessed and this system will detect the cancerous nodules. In the proposed system Krill Herd Optimization algorithm is used to find the optimum threshold values by maximizing Otsu's or Kapur's objective function and the Artificial Neural Network is used to detect the cancerous nodules. This technique will assist radiologists to diagnose the lung cancer in the earlier stages.
\end{abstract}

Keywords: Image Segmentation, Krill Herd Optimization, Artificial Neural Networks, multilevel thresholding.

\section{INTRODUCTION}

Lung Cancer is considered to be the most grave health condition, and the lung cancer patient rate of survival is very less even though the cancer is detected, because in most of the cases cancer is diagnosed in the later stages, So to improve the life expectancy of the lung cancer patient earlier diagnosis of the lung cancer is a must. Lot of research is going on in this area, and simultaneously rate of lung cancer casualty is also growing year by year as per the statistics almost $90 \%$ of the male and $80 \%$ of female are predicted with lung cancer due to smoking of cigarette [1]

There are two main categories of lung cancer namely nonsmall cell and small cell and there are 4 different stages of lung cancer and these stages are identified based on the characteristics like size of the nodule and location of lymph node. The rate of survival depends on at what stage cancer cells are discovered.

The first operation which is used to analyze the acquired image is image segmentation, it's a process of
Segmenting an image, among the various image segmentation techniques thresholding offers better accuracy, robustness and simplicity. Among many thresholding techniques available in the literature Otsu's method and kapur's methods are the two best proven techniques. To solve multilevel thresholding here we present a new technique called Krill Herding Optimization Algorithm [2].

\section{THE METHODOLOGY}

Proposed lung cancer detection system will be consisting of enhancing the image, process of feature extraction, segmentation and classification. The frame work is shown in the following figure 1

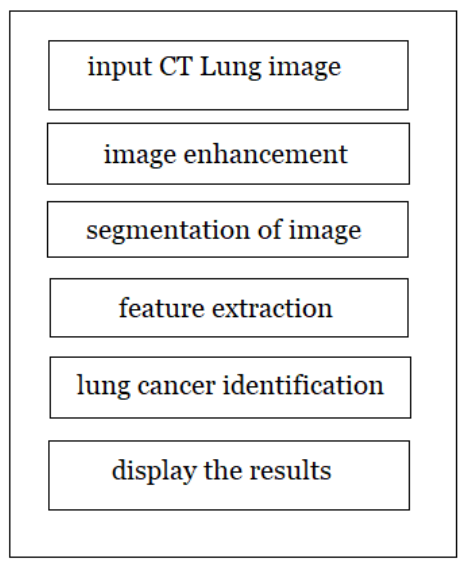

Figure 1: overall frame work for lung cancer detection system

\subsection{Input CT Lung Image}

The first step is to collect the lung CT image from LIDCIDRI(LUNG IMAGE DATABASE CONSORTIUM and IMAGE DATABASE RESOURCE INITIATIVE)[3][8]

\subsection{Image Enhancement}

To enhance the CT lung input images Adaptive equalization of histogram technique is used, which enhances the image contrast and best suited for improving the images by eliminating noise at the pixel level [9][10] asshown in figure 2. 
The process of enhancing each pixel is done with the help of histogram equalization and if noise amplifies and artifacts occurs in the boundary region $[10,12]$ of an image then contrast limited adaptive histogram equalization is applied

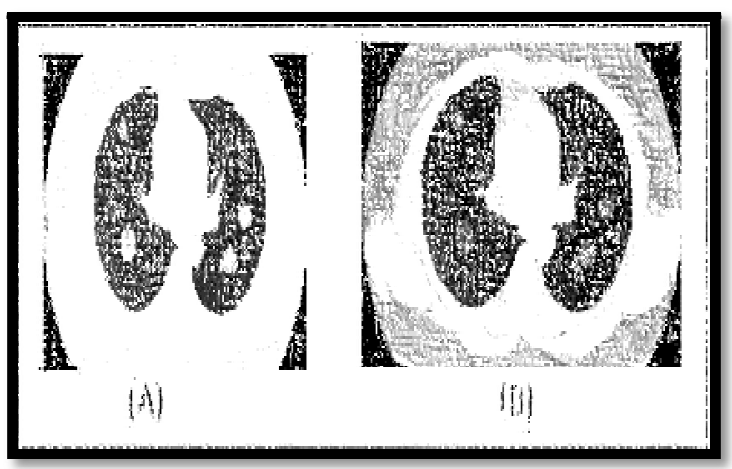

Figure 2: (A) Original Image (B) Original Image with Histogram Equalization

\subsection{Image Segmentation}

\section{Multilevel Thresholding}

Segmenting a gray level image and generating many different regions out of it can be done with the helpof Multilevel Thresholding. Using more than on thresholding technique to segment the image into bright regions of which one will be background and rest will be objects.

Here in this work, Fitness function for the Krill Herd algorithm is proposed on two criteria's entropy criterion and between class variance.

\section{Krill Herd Optimization Algorithm}

KHO Algorithm [2] is a novel swam intelligent technique which simulates herding behavior of the krill's individually. Fitness function of the krill's individually is used to identify the centers for food.Each krill position can be identified depending on Movement induction(Nki), random diffusion(Dki) and foraging activity(Fki) of the individual krill. The Block Diagram of KHO based image segmentationis shown in the Figure 3.

\section{Algorithm}

1) Initialize the parameters $I_{\max }$ (max. iteration), $\mathrm{N}^{\max }$ (maximum induced speed), $\mathrm{V}_{\mathrm{f}}$ (maximum foraging

(number of speed), $\mathrm{D}^{\max }$ (maximum diffusion speed), $\mathrm{n}$ Krill) and X (position of Krill).

2)Repeat Step 3 to Step7 for $\mathrm{i}=1$ to $\mathrm{I}_{\max }$

3) For each Krill calculate movement

4) Movement due to other Krill individuals
$\mathrm{Nk}_{\mathrm{i}}^{\text {new }}=\mathrm{N}^{\mathrm{max}} \phi_{\mathrm{i}}+\omega_{\mathrm{n}} \mathrm{Nk}_{\mathrm{i}}^{\text {old }}$

$\omega_{n}$ is the inertia weight associated with the motion induced and has a value in the range $[0,1]$. $N k_{i}^{\text {old }}$ represents the last motion indurad

\section{Step 4.1}

$\phi_{i}=\phi_{i}^{\text {local }}+\phi_{i}^{\text {target }}$

Step 4.1.1 The neighbors provides a local effect denoted by $\phi_{i}^{\text {local }}$ and it can be defined as follows:

$\phi_{i}^{\text {local }}=\sum_{j=1}^{N n} \hat{K}_{i, j} \hat{X}_{i, j}$

where,

$\hat{X}_{i, j}=\frac{X_{j}-X_{i}}{\left\|X_{j}-X_{i}\right\|+z}$

$\hat{K}_{i j}=\frac{K_{i}-K_{j}}{K^{\text {Worst }}-K^{\text {best }}}$

$\mathrm{K}^{\text {worst }}$ and $\mathrm{K}^{\text {best }}$ individual krill's worst and best fitness values.

Step 4.1.2 Effect of target direction denoted by $\phi_{i}^{\text {target }}$ is provided by the best Krill individual and is determined as follows

$\phi_{i}^{\text {target }}=C^{\text {best }} \widehat{K}_{i, \text { best }} \hat{X}_{\text {ibest }}$

Krill individual effective coefficient with best fitness to $i^{\text {th }}$ krill individual denoted by $C^{\text {best }}$ is given by,

$C^{\text {best }}=2\left(\right.$ rand $\left.+\frac{l}{I_{\max }}\right)$

Step 4.2 Compute sensing distance

$$
d_{s, i}=\frac{1}{5 N} \sum_{j=1}^{N} X_{i}-X_{j}
$$

5) Movement due to foraging activity

$$
\mathrm{Fk}_{\mathrm{i}}=\mathrm{V}_{\mathrm{f}} \gamma_{\mathrm{i}}+\omega_{\mathrm{f}} \mathrm{Fk}_{\mathrm{i}}^{\text {old }}
$$

6) Physical Diffusion

\section{7)Genetic Operators}

Figure 4 shows three images where the first image is an input image Figure 4.a) and the Figure 4.b) is a Filtered Image Figure 4.c) is a Segmented Image. 


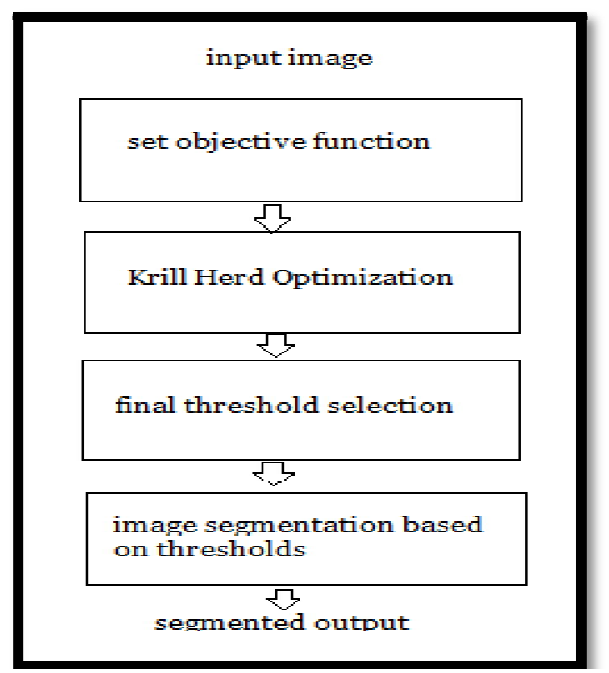

Figure 3: Blockdiagram for KHO based image segmentation

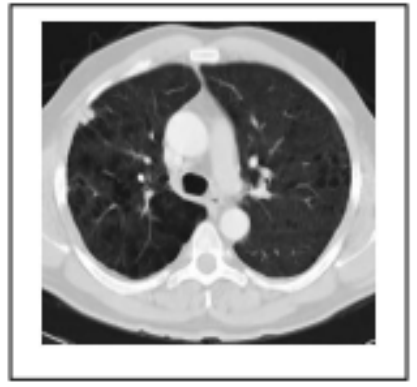

(a)

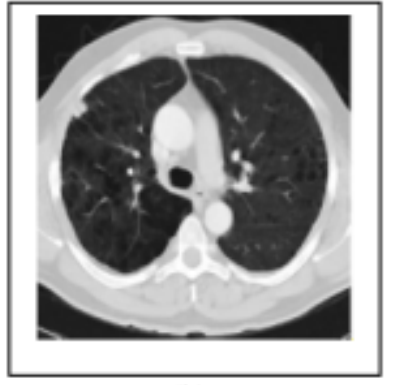

(b)

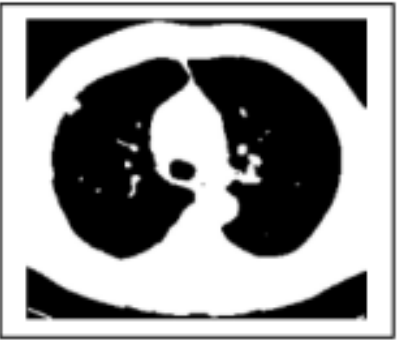

(c)

Figure 4: a) CT Image b) Filtered Image c) Segmented Image

\subsection{Feature Extraction}

Much of texture data will be present in the lung image which is used for determining the unique parts in the image. To match the image database lung image patterns must be extracted in a noiseless process which will increase the quality of matching, which can be accomplished by making use of 5HAAR wavelet transform which decomposes the image.

The steps to follow are

Step 1: apply haar's two-dimensional DWT on the input lung image till 5-level decomposition is achieved.

Step 2: extract the features of the lung image in terms of vectors which was provided during level 4 and 5.

Step 3: feature vectors will be in the binary form.
Step 4: save the binary code.

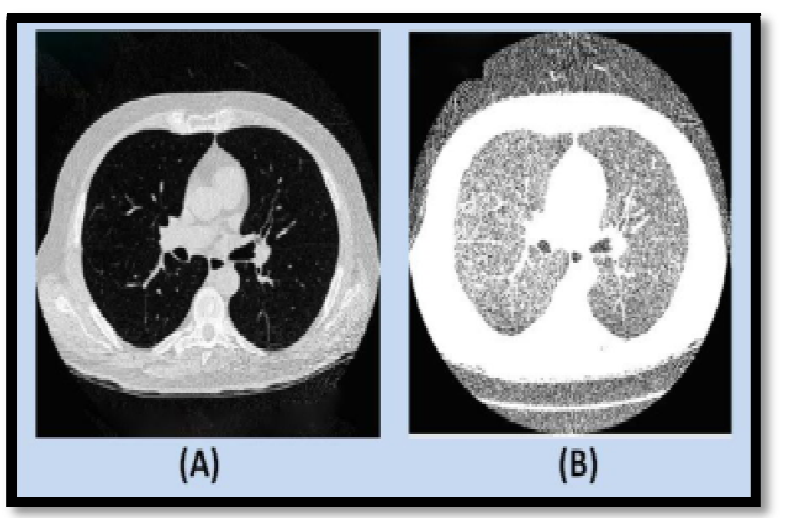

Figure 5: a) Original Image b) Enhanced image

Figure 5.a) shows the Original Image and the enhanced image is shown in Figure 5.b)

\subsection{Lung Cancer Detection}

\section{Artificial Neural Network Based Detection}

Network is developed and image Feature vectors are givento the ANN as input and the ANN is trained by means of already known targets using FFNN. Output of the trained network will be tested by assessing its result with the expected results [13]-[19].

\subsection{Results}

MATLAB is used to design this work and LIDC open repository is the source for lung images and Figure 6.a) shows Original image and Figure 6.b) shows the masked image, Figure 6.c) shows Enhanced image and Figure 6.d) shows Bordered Image and finally Figure 6.e) shows the image with cancer location.

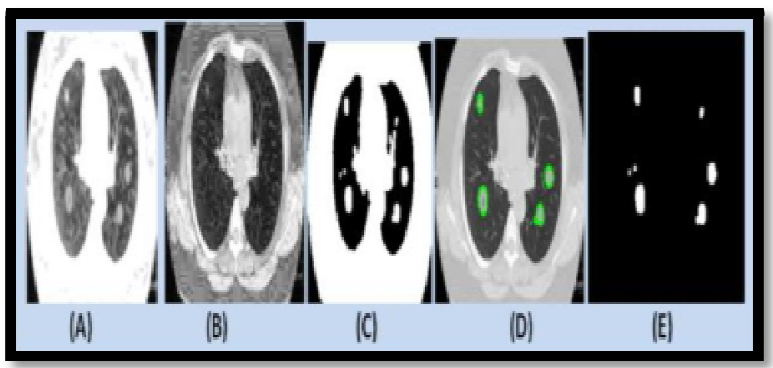

Figure 6: a) Original Image b) Masked Image c) Enhanced Image d)Bordered Image e) Cancer location is predicted

\section{CONCLUSION}

With the help of image processing tumor detection on CT lung images is carried out, which enhances the input image quality for possible earlier detection of cancer. The proposed method uses a novel Krill Herd Optimization Algorithm for image segmentation and ANN for detection of the cancer. 
For earlier detection of cancer on the CT lung images image is enhanced using five level HAAR wavelet transform technique and KHO Algorithm (Krill Herd Optimization Algorithm) for multilevel thresholding is used. This method detects the chances of lung cancer occurrence more precisely.

\section{REFERENCES}

[1] A. Jemal., T. Murray, E. Ward. , A. Samuels., R. C. Tiwari., A.Ghafoor.E. J. Feuer., and M. J. Thun., "Cancer statistics", CA: acancer journal for clinicians, Vol. 55, No 1, pp.10-30, 2005.

https://doi.org/10.3322/canjclin.55.1.10

[2] Gandomi, Amir Hossein, Alavi, Amir Hossein, 2012. Krill Herd: a new bio-inspired optimization algorithm. Commun. Nonlinear Sci. Numer. Simulat. 17, 4831-4845. https://doi.org/10.1016/j.cnsns.2012.05.010

[3] R. Raghavendra, R. Ashok, and G. H. Kumar, "Multimodal biometric score fusion using Gaussian mixture model and Monte Carlo method", Journal of Computer Science and Technology, Vol.25, No. 4, pp. 771-782, 2010.

[4] S. J. Xie., J. Yang., D. S. Park., S. Yoon., and J. Shin., "State of the art in biometrics", Iris Biometric Cryptosystems, InTech, 2011.

[5] H. Zhang, Y. Zhu, W. Zou, X. Yan, "A hybrid multiobjective artificial bee colony algorithm for burdening optimization of copper strip production", Applied Mathematical Modelling, Vol. 36, No. 6,pp: 2578-2591, 2012.https://doi.org/10.1016/j.apm.2011.09.041

[6] X. Liao, J. Zhou, R. Zhang, Y. Zhang, "An adaptive artificial bee colony algorithm for long-term economic dispatch in cascaded hydropower systems", International Journal of Electrical Power and Energy Systems, Vol. 43, No. 1, pp: 1340-1345, 2012.

[7] S. Sivakumar, and C. Chandrasekar, "Feature Selection Using ABC for the Lung CT Scan Images", International Journal of Scientific Engineering and Technology, Vol: 3, No. 6, pp: 781-784, 2014.

[8] W. Zou., Y. Zhu., H. Chen., Z. Zhu., "Cooperative approaches to artificial bee colony algorithm" Computer Application and System Modeling (ICCASM), Vol. 9, pp. V9-44). 2010.

[9] N. Suguna and K. G. Thanushkodi, "An Independent Rough Set Approach Hybrid with Artificial Bee Colony Algorithm for Dimensionality Reduction", American Journal of Applied Sciences 8 (3): 261 - 266, 2011.

https://doi.org/10.3844/ajassp.2011.261.266

[10] Li Bao and Jian-chao Zeng, Comparison and Analysis of the Selection Mechanism in the Artificial Bee Colony Algorithm, Proc. IEEE Ninth International Conference on Hybrid Intelligent Systems, pp: 411-416, 2009.

[11] S. J. Xie., J. Yang., D. S. Park., S. Yoon., and J. Shin., "State of the art in biometrics", Iris Biometric Cryptosystems, InTech, 2011.

[12] H. Zhang, Y. Zhu, W. Zou, X. Yan, "A hybrid multiobjective artificial bee colony algorithm for burdening optimization of copper strip production", Applied
Mathematical Modelling, Vol. 36, No. 6, pp: 2578-2591, 2012.

[13] S.Ramkumar ,K.Sathesh Kumar, T.DhiliphanRajkumar, M.Ilayaraja, K.Shankar, "A review-classification of electrooculogram based human computer interfaces", Biomedical Research, 29(6), Pp. 1078-1084, April 2018.

[14] Hema.C.R, Paulraj.M.P\&Ramkumar.S, "Classification of Eye Movements Using Electrooculography and Neural Networks", International Journal of Human Computer Interaction, Vol.5 (4), pp.51-63, 2014.

[15] Hema, C. R., Ramkumar, S., \&Paulraj, M. P. , "Idendifying Eye Movements using Neural Networks for Human Computer Interaction", International Journal of Computer Applications, 105(8), pp 18-26, 2014.

[16] S.Ramkumar, K.SatheshKumar, G.Emayavaramban, "EOG Signal Classification Using Neural Network for Human Computer Interaction", International Journal of Computer Theory and Applications, Vol.9(24) , pp.223-231, 2016

[17] Ramkumar, Dr.K.Satheshkumar and G.Emayavaramban" Nine States HCI using Electrooculogram and Neural Networks", IJET, Vol. 8(6), pp. 3056-3064, Jan 2017.

https://doi.org/10.21817/ijet/2016/v8i6/160806249

[18] S.Ramkumar, K.Sathesh Kumar G.Emayavaramban," A Feasibility Study on Eye Movements Using Electrooculogram Based HCI" IEEE- International Conference on Intelligent Sustainable Systems, pp.384-388, Dec-2017.

https://doi.org/10.1109/ISS1.2017.8389435

[19] G.Emayavaramban, S.Ramkumar, A.Amudha and K.Sathesh Kumar "Classification Of Hand Gestures Using FFNN And TDNN Networks", International Journal of Pure And Applied Mathematics, Vol.118 (8) Pp. 27-32, Jan 2018. 